\title{
Treat to Target in the Management of Chronic Uveitis
}

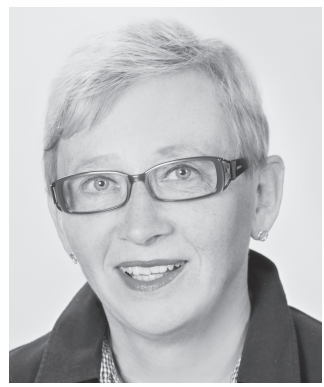

Uveitis is a cause of visual loss in $10 \%$ of the visually handicapped. The etiology of uveitis remains unknown in about $50 \%$ of cases. The most important diseases behind endogenous uveitis are spondyloarthropathies, juvenile idiopathic arthritis (JIA), sarcoidosis, multiple sclerosis, and tubulointerstitial nephritis. Uveitis may be caused by infectious agents such as toxoplasma, herpes viruses, Borrelia burgdorferi, cytomegaloviruses, tuberculosis, and syphilis. If the etiology is known, the treatment strategy is according to the underlying disease ${ }^{1,2,3}$.

The treatment of chronic endogenous uveitis is frequently difficult. The first-line standard therapy for uveitis is topical, periocular, and systemic corticosteroids; and often methotrexate (MTX), as a second-line disease-modifying antirheumatic drug (DMARD). Tumor necrosis factor- $\alpha$ (TNF- $\alpha$ ) blockers have been used to treat uveitis associated with JIA in children who have failed conventional topical and immunosuppressive therapy. Infliximab and adalimumab, in particular, have been shown to be effective for chronic uveitis, frequently in combination with MTX, to increase the treatment effect and to decrease the risk of development of neutralizing antibodies against the TNF-blocker. If MTX is not tolerated or is ineffective, azathioprine, leflunomide, cyclosporin A, or mycophenolate mofetil may be used. Other biologic drugs, such as abatacept, tocilizumab, rituximab, and golimumab, may be beneficial in severe uveitis ${ }^{4,5}$.

In this issue of The Journal, Pelegrin, et $a l^{6}$ report promising results of rituximab treatment in 9 patients with uveitis who were refractory to standard immunosuppressive treatment. Rituximab was effective and induced clinical remission of uveitis in most patients.

Treatment experiences of DMARD combination are also described in this issue for treatment of childhood uveitis. Little, et $a l^{7}$ examined the effect of combining a third immunosuppressive drug with the conventional immunosuppressive treatment, which mainly consisted of MTX and
TNF- $\alpha$ blocker. In their 13 uveitis patients, triple therapy did not confer substantial benefit for chronic uveitis; instead the therapy was associated with increased risk of infectious adverse effects.

The treatment of chronic uveitis is challenging. The model of the treat-to-target approach in the management of JIA may also be suitable for the treatment of uveitis: early and aggressive treatment of uveitis to achieve clinical remission and even disease inactivation ${ }^{8}$. This could be accomplished by strict monitoring of patients with uveitis by uveitis specialists, in close cooperation with rheumatologists, pediatric rheumatologists, internists, and immunologists.

In the near future, more reports of uveitis treatment with new biologic drugs are anticipated; hopefully, prospective, comparative, controlled studies to manage this severe disease will mean even better results than today. Despite the sometimes high costs of drugs, the basic goal of treatment is to prevent blindness in these patients.

KAISU KOTANIEMI, $\mathrm{MD}, \mathrm{PhD}$, Department of Ophthalmology, Helsinki University Hospital, Helsinki, Finland.

Address correspondence to Dr. Kotaniemi.

E-mail: kaisumkotaniemi@gmail.com

\section{REFERENCES}

1. Kotaniemi K, Aho K, Kotaniemi A. Uveitis as a cause of visual loss in arthritides and comparable conditions. J Rheumatol 2001;28:309-12.

2. Kanski JJ. Anterior uveitis in juvenile rheumatoid arthritis. Arch Ophthalmol 1997;95:1794-7.

3. Smith JA, Mackensen F, Sen NH, Leigh JF, Watkins AS, Pyatetsky $\mathrm{D}$, et al. Epidemiology and course of disease in childhood uveitis. Ophthalmology 2009;116:1544-51.

4. Heiligenhaus A, Michels H, Schumacher C, Kopp I, Neudorf U, Niehues T, et al. Evidence based, interdisciplinary guidelines for anti-inflammatory treatment of uveitis associated with juvenile idiopathic arthritis. Rheumatol Int 2012;32:1121-33.

See Rituximab for ocular autoimmune diseases, page 84 and Triple therapy in pediatric uveitis, page 136 
5. Simonini G, Cantarini L, Bresci C, Lorusso M, Galeazzi M, Cimaz R. Current therapeutic approaches to autoimmune chronic uveitis in children. Autoimmun Rev 2010;9:674-83.

6. Pelegrin L, Jakob E, Schmidt-Bacher A, Schwenger V, Becker M, Max R, et al. Experiences with rituximab for the treatment of autoimmune diseases with ocular involvement. J Rheumatol 2014;41:84-90.

7. Little JA, Sen ES, Strike H, Hinchcliffe A, Guly M, Lee RWJ. The safety and efficacy of noncorticosteroid triple immunosuppressive therapy in the treatment of refractory chronic noninfectious uveitis in childhood. J Rheumatol 2014;41:136-9.

8. Consolaro A, Negro G, Lanni S, Solari N, Martini A, Ravelli A. Toward a treat-to-target approach in the management of juvenile idiopathic arthritis. Clin Exp Rheumatol 2012;30 Suppl 73: S157-S162.

J Rheumatol 2014;41:3-4; doi:10.3899/jrheum.131148 